\title{
Capsulation gene loss and 'rescue' mutations during the Cap' to Cap'- transition in Haemophilus influenzae type b
}

\author{
Lisa N. Brophy, ${ }^{1 *}$ J. Simon Kroll, ${ }^{1}$ David J. P. Ferguson ${ }^{2}$ and E. Richard Moxon ${ }^{1}$ \\ ${ }^{1}$ Institute of Molecular Medicine, Oxford University, John Radcliffe Hospital, Oxford OX3 9DU, UK \\ ${ }^{2}$ Nuffield Department of Pathology, John Radcliffe Hospital, Oxford OX3 9DU, UK
}

(Received 7 May 1991; accepted 22 July 1991)

\begin{abstract}
Genes for Haemophilus influenzae type b capsule expression are duplicated to form a potentially unstable structure, cap, of directly-repeated chromosomal regions of approximately $17 \mathrm{~kb}$. Capsule-deficient mutants arise in a two-stage process, initiated by rec-dependent reduction of this region from two copies to one. This recombinational event is usually lethal, only about $1 / 200$ surviving to form slow-growing colonies of organisms that continue to synthesize polysaccharide but are defective in its export. A variety of secondary 'rescue' mutations within cap can occur to reduce polysaccharide synthesis and restore normal organism appearance and colony morphology.
\end{abstract}

\section{Introduction}

Haemophilus influenzae causes bacterial meningitis and other life-threatening invasive infections in young children. More than $95 \%$ of systemic infections caused by $H$. influenzae are due to organisms possessing the type b capsule (Turk, 1982), a polymer of ribose and ribitol 5phosphate (PRP), which is an important virulence determinant (Moxon et al., 1984).

It has long been recognized that PRP synthesis and type $\mathrm{b}$ capsule expression is readily lost by type $\mathrm{b}$ strains (Fothergill \& Chandler, 1936). This commonly occurs in two steps (Buckmire, 1976). The first event gives rise to the class I mutant, which appears at a frequency of $0 \cdot 1-0.5 \%$ (Hoiseth et al., 1985). It is easily distinguished from its capsulate parent as organisms are highly pleomorphic and frequently filamentous, forming small, rough, non-iridescent colonies (see Fig. 1). A second event gives rise to class II variants, in which PRP synthesis is virtually abolished (Buckmire, 1982). Colonies of class II variants are large and smooth but noniridescent on inspection by obliquely transmitted light (Fig. 1) and contain cocco-bacillary organisms of uniform appearance.

A region of the $H$. influenzae type b genome, the cap locus, is critically involved in capsule expression. This locus contains two directly repeated $17 \mathrm{~kb}$ DNA seg-

Abbreviations: $\mathrm{b}^{+}$, type b capsulate; (s)BHI, (supplemented) brainheart infusion; cap, capsulation locus; FITC, fluorescein isothiocyanate; PRP, poly-ribosyl ribitol phosphate. ments separated by a unique region of approximately $1.2 \mathrm{~kb}$ - the bridge region (Hoiseth et al., 1986; Kroll et $a l ., 1988)$. When EcoRI digests of total genomic DNA of either class I or class II variants are probed with pSKH2, containing DNA cloned from the cap locus (Hoiseth et al., 1985), a $9 \mathrm{~kb}$ band seen in the capsulate $\left(\mathrm{b}^{+}\right)$parent strain is lost. Otherwise the probing patterns are identical. This change in probe hybridization has been shown to correspond to reduction of cap to a single copy the residual cap locus - through recombination between the direct repeats (Hoiseth et al., 1986). In this rearrangement, the $9 \mathrm{~kb} E c o \mathrm{RI}$ fragment spanning the junction between the cap repeats is lost, and with it the $1.2 \mathrm{~kb}$ bridge region (Fig. 2). The phenotype of class I variants can be reproduced by site-directed mutagenesis in the bridge region. This disrupts the gene bexA which has been shown to play a critical role in polysaccharide export (Kroll et al., 1988) and supports the proposition that the defect in the spontaneous class I variants is in polysaccharide export rather than synthesis (Catlin \& Tartagni, 1969; Buckmire, 1982). It has been suggested that intracellular accumulation of polysaccharide is responsible for the 'unhealthy' phenotype (Kroll et al., 1988).

Using direct light and immunofluorescence microscopy with FITC-conjugated anti-PRP antibody (Buckmire, 1982; Catlin \& Tartagni, 1969), class I and class II variants are readily distinguished from one another by the observation that the former are pleomorphic, with irregular accretions of intracellular capsular material, 
while the latter are uniform cocco-bacilli making very little or no PRP. The identity of cap probing pattern between class I and class II variants suggests that no large-scale rearrangement at the cap locus accompanies the change in phenotype. The change could come about through the down-regulation of PRP synthesis, with the implication of reversibility, or through mutation, either in biosynthetic genes within cap or in some other chromosomal locus altogether. In eight independent class II variants of the prototypic type b strain Eagan, we have found that the transition from class I to class II phenotype was the result of mutation in cap genes.

\section{Methods}

Bacterial strains. H. influenzae RM153 (Eagan) is a prototypic virulent type b strain (Anderson et al., 1972). Eagan and its variants described in this work were grown in brain-heart infusion broth

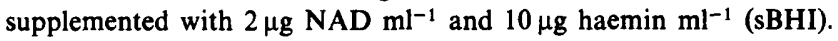
BHI plates were prepared with $1 \%(w / v)$ agar supplemented with $10 \%$ (v/v) Levinthal base (Alexander \& Leidy, 1965) and strains were stored at $-70^{\circ} \mathrm{C}$ in sBHI broth and $20 \%(\mathrm{v} / \mathrm{v})$ glycerol.

Phenotypic characterization of $H$. influenzae. Diluted cultures of $H$. influenzae were plated on to translucent sBHI agar and colony phenotype was assessed by viewing with obliquely-transmitted light as previously described (Hoiseth et al., 1985). Immunofluorescent microscopy using rabbit anti-PRP antibody conjugated to FITC (Catlin \& Tartagni, 1969) was used to assess the surface distribution of capsular antigen. Electron microscopy was performed as described previously (Kroll et al., 1988).

Transformation of $H$. influenzae. H. influenzae was made competent for the uptake of linearized DNA by growth in SBHI broth followed by incubation in MIV medium (Herriott et al., 1970) as described previously (Moxon et al., 1984).

Preparation and analysis of $H$. influenzae chromosomal DNA. Total cellular DNA was prepared from $3 \mathrm{ml}$ broth cultures as previously described (Moxon et al., 1984). Genetic analysis of variants and transformants was accomplished by digestion of total cellular DNA with EcoRI, electrophoresis in agarose/Tris acetate gels and Southern blotting. The probe pUO38, containing a $18.2 \mathrm{~kb} \mathrm{BamHI}$ fragment from cap (Kroll et al., 1988; and Fig. 2) was used to construct restriction maps of the residual cap locus in each variant.

Transforming DNA. Plasmids used for transformation contain EcoRI fragments from the cap locus cloned into the EcoRI site of pBR328, the name corresponding to the size of each fragment, and the plasmids pUO38 and pUO12 (Fig. 2).

\section{Results}

\section{Independent class II variants can be transformed to the class I and capsulate phenotypes with cap DNA}

A single colony of strain Eagan was grown to midexponential phase in $\mathrm{sBHI}$ and plated on to $\mathrm{sBHI}$ agar at a density of approximately 1000 colonies per plate. Eight class I colonies were selected by their characteristic appearance and separately streaked on to $\mathrm{SBHI}$ agar to produce spontaneous class II variants (Fig. 1), and then one class II variant per plate was picked. This ensured that all the class II variants that formed the basis of this study arose through genetically independent events. The genotype of each class I and class II variant was checked by Southern blotting (Fig. 2). All were identical, with a residual cap locus containing only a single copy of the $\sim 17 \mathrm{~kb}$ segment duplicated in the capsulate parent (data not shown). The class II variants were made competent for uptake of linearized DNA and exposed to the $18.2 \mathrm{~kb}$ BamHI cap fragment excised from pUO38, seeking the class I phenotype in the products of transformation. In each case not only class I variants but also capsulate organisms were recovered. Transformants appeared identical to wild-type class I variants and capsulate organisms by several criteria: (i) colonial appearance; (ii) microscopic appearance after Gram and immunofluorescence staining (Fig. 1) and (iii) Southern blotting using pUO38 as a probe. Class I transformants had a probing pattern identical to the class II strains from which they were derived (Fig. 2). In contrast, capsulate transformants were found to have regained the $9 \mathrm{~kb}$ EcoRI fragment containing the bridge region, suggesting that the duplicated cap locus had been regenerated. The possibility of an appreciable rate of reversion of class II to class I phenotypes was refuted by examination of cultures of class II variants plated at low density on to sBHI agar. Approximately 20000 colonies were screened but none with the class I or capsulate phenotype was seen. The process of induction of competence for DNA uptake similarly does not induce such a phenotypic change.

\section{Class II mutations are not confined to a single 'hotspot' in cap}

To determine whether spontaneous mutations leading to the class II phenotype were localized to a 'hotspot' in cap, an attempt was made to transform the eight independent class II strains as before, but now using smaller nonoverlapping fragments of cap DNA. In all cases the efficiency of transformation was found to be substantially reduced. Each strain could only be transformed by, at most, one of these fragments, and different fragments were necessary in each case (Table 1). Thus the secondary mutations leading to the class II phenotype do not appear to be limited to a single 'hotspot' but can occur at different locations throughout cap.

\section{Recombinational reduction of cap to the class I genotype is often lethal}

Recombinational events between adjacent identical $\sim 17 \mathrm{~kb}$ segments of DNA would be expected to occur at 
(a)
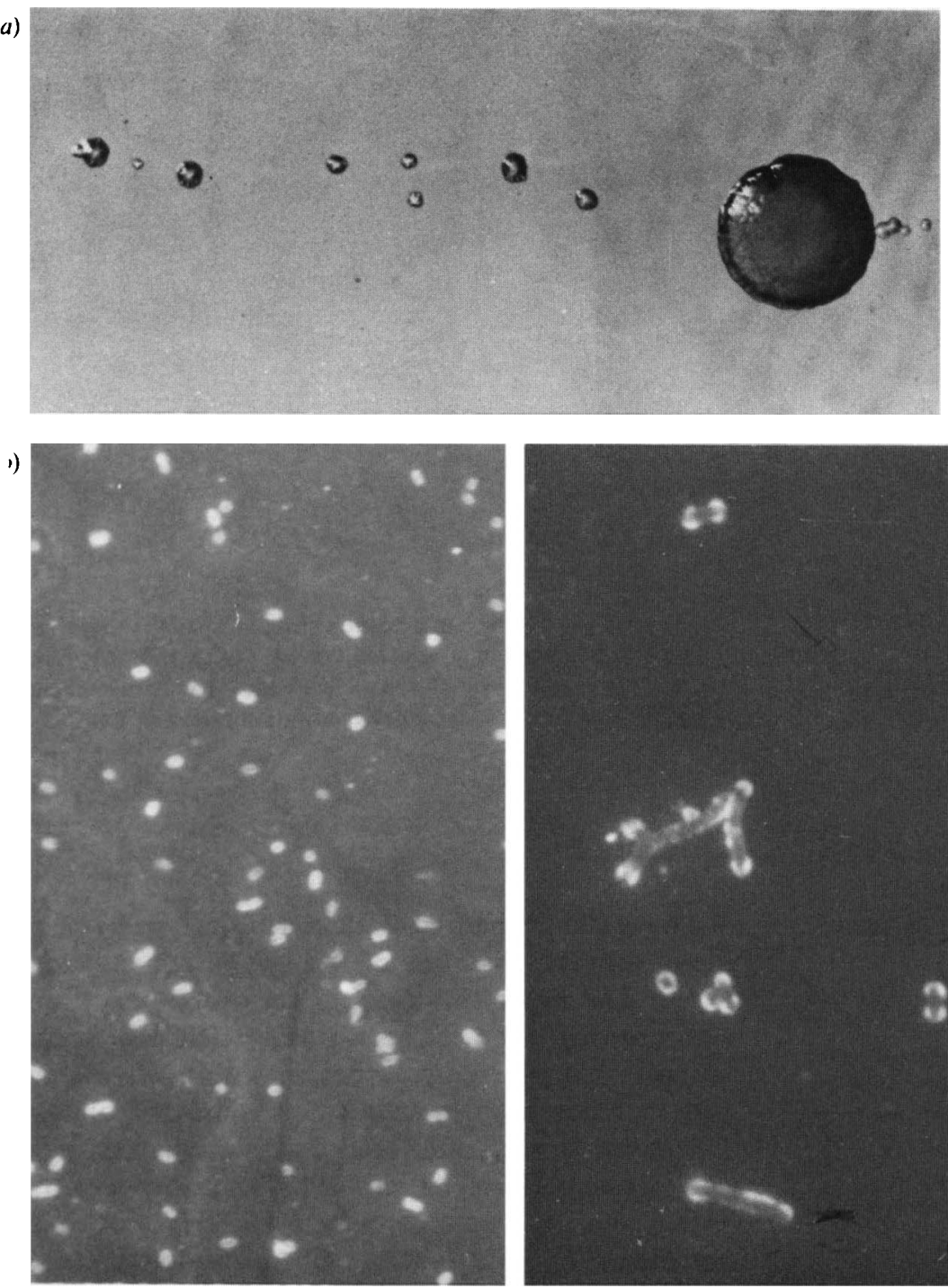

$b^{+}$

(a)

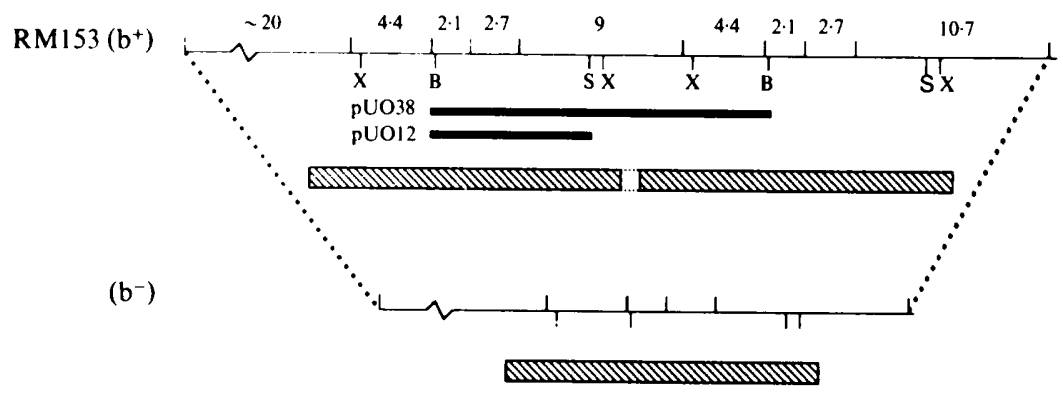

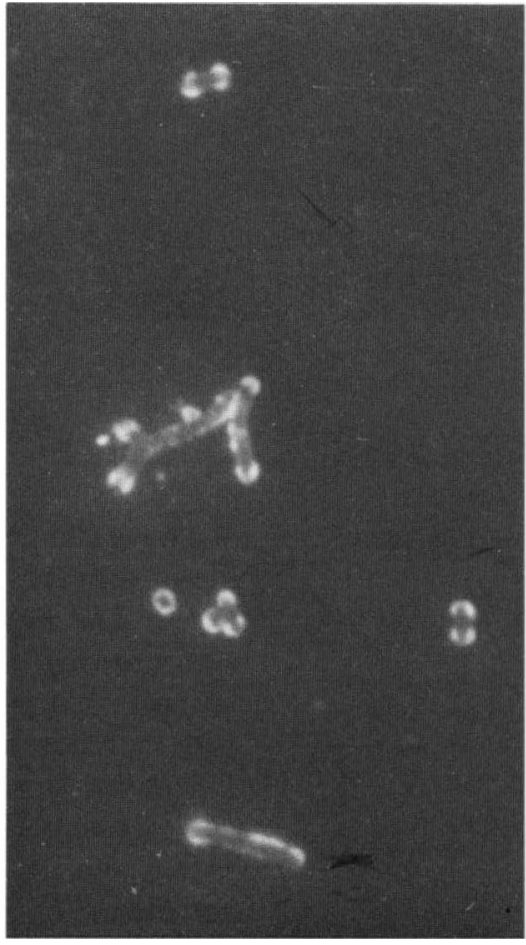

Class I
Fig. 1. (a) Class I (tiny) and a spontaneous class II (large) mutant colony viewed by obliquely-transmitted light. (b) Immunofluorescent photomicrographs showing cocco-bacillary type b organisms ringed with fluorescence, class I organisms showing irregular and bipolar distribution of immunoreactive material, and class II organisms containing little or no immunoreactive material.

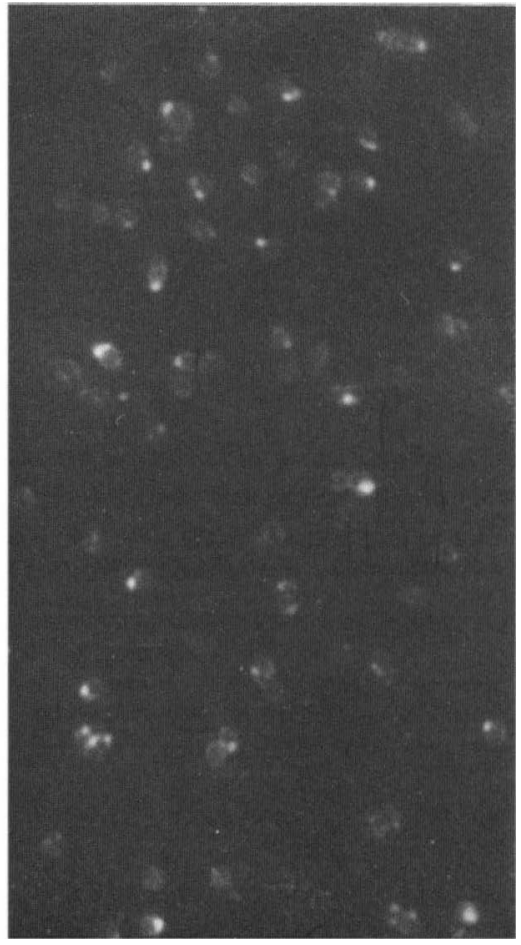

Class II

(b)

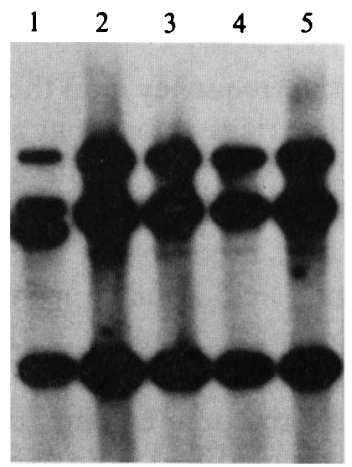

Fig. 2. (a) Physical map of the cap locus of the type b strain RM153. EcoRI sites are represented by the unlabelled vertical lines above the chromosome. Other restriction sites: X, XhoI; S, SalI; B, BamHI. Hatched bars represent the cap repeats. Black bars show the DNA contained in the plasmids pUO38 and pUO12. Recombinational reduction of the locus is also indicated. (b) Southern blot of EcoRI fragments of chromosomal DNA of $b^{+}$(track 1), class I (tracks 2,4) and class II (tracks 3,5) strains probed with nick-translated pUO38 showing the loss of the $9 \mathrm{~kb} E c o$ RI fragment in the class I mutant and no difference in the probing patterns of class I and class II mutants (only fragments over $4 \mathrm{~kb}$ are shown). 


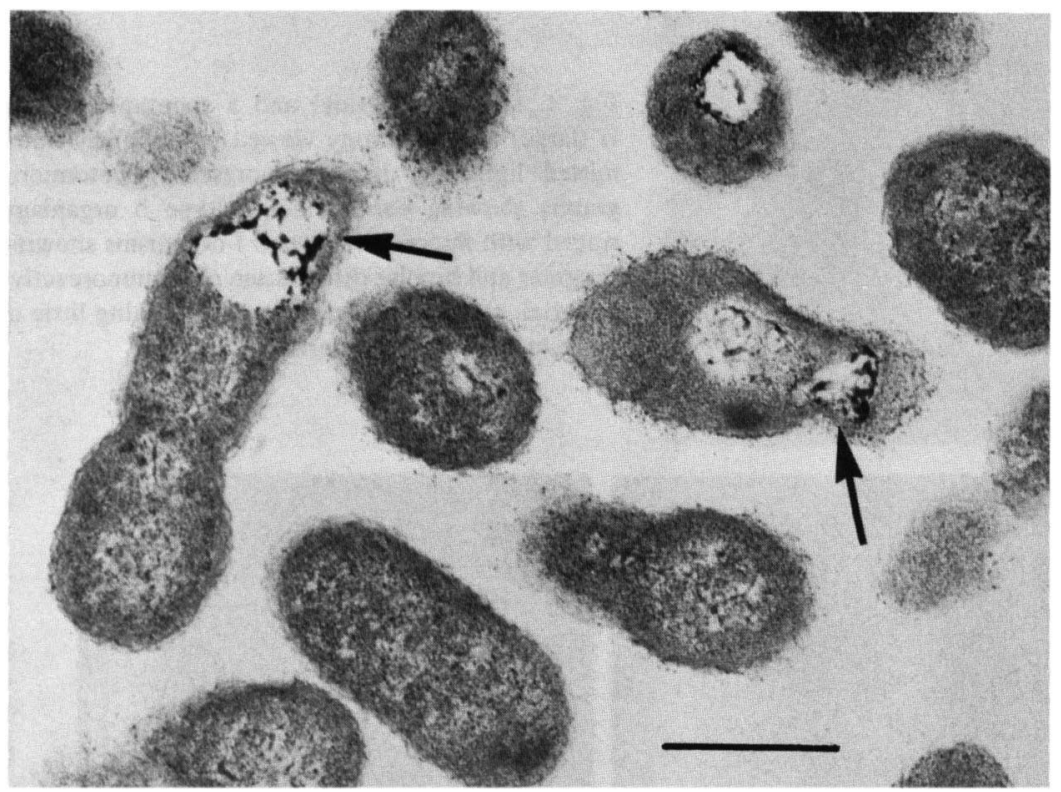

Fig. 3. Transmission electron micrograph through a randomly selected colony of RM153 showing the presence of spontaneous class I mutants characterized by terminal vacuolation (arrows). Bar, $0.5 \mu \mathrm{m}$.
Table 1. Effect of using different fragments of cap DNA to transform independently isolated class II variants

$(+)$ indicates that transformants were obtained; $(-)$ indicates that no transformants were observed; ND, not done.

\begin{tabular}{ccccccc}
\hline \hline & \multicolumn{7}{c}{ Transforming DNA: } \\
\cline { 2 - 7 } Strain & pUO38 & pUO12 & $9 \mathrm{~kb}$ & $4.4 \mathrm{~kb}$ & $2 \cdot 7 \mathrm{~kb}$ & $2 \cdot 1 \mathrm{~kb}$ \\
\hline 5566 & + & ND & + & - & - & - \\
5567 & + & + & - & - & + & - \\
5569 & + & ND & - & + & - & - \\
5570 & + & - & - & - & - & - \\
5571 & + & - & - & - & - & - \\
5574 & + & - & - & - & - & - \\
5576 & + & + & - & - & - & - \\
5577 & + & - & - & - & - & - \\
\hline \hline
\end{tabular}

a substantial frequency, and the occurrence of spontaneous class I variant colonies at a rate of only about $0.5 \%$ from cultures of type $b$ strains suggested that organisms with the residual cap genotype might be of reduced viability. This is consistent with the observation of slow growth and 'sick' appearance of organisms making up class I colonies, choked with intracellular capsular material (Kroll et al., 1988). This was tested by direct electron microscopic examination of dispersed spreads of organisms obtained from exponential cultures of type b strains, seeking the vacuolated export-defective class I variant phenotype at the organism level (Kroll et al., 1988) (Fig. 3). In blind screening, 91 of 450 Eagan organisms were vacuolated $(20 \%)$, in contrast to spreads of class II variants, in which no vacuolation was seen.

\section{Discussion}

We have shown that the reduction of the type b cap locus direct repeats, with loss of the bridge region, is often lethal. The organisms that do survive produce small, slow-growing colonies. This is associated with accumulation of capsular polysaccharide within the organism and that has been speculated to cause the abnormal phenotype through interference with normal cell growth and division (Kroll et al., 1988). Further mutations within the cap locus of class I bacteria give rise to organisms of the healthy, unencapsulated class II phenotype. These organisms no longer accumulate intracellular polysaccharide, suggesting that mutations have impaired its synthesis. All the examples of these mutations studied here could be corrected by transformation with cloned cap DNA from a $\mathrm{b}^{+}$strain to produce class I organisms again. This strongly suggests that the class I to class II transition is not regulated by genes outside the cap locus. Independently derived class II strains have mutations in different parts of their residual cap locus since examples can be transformed to class I by different cloned segments. There appears, therefore, to be no single 'hotspot' for secondary mutations within cap.

The electron microscopic quantification suggests that in an exponential culture of a type $b$ strain, about $20 \%$ of organisms have the class I phenotype. However, only about $0.5 \%$ are detectable at the colony level; $99.5 \%$ of class I organisms die. The mutations leading to the class II colonial phenotype can thus be seen as 'rescue' mutations. The cessation of synthesis of capsular 
material by any of a number of mutations within cap can apparently restore normal cellular appearance and growth rate.

The duplication of cap genes generally found in type b strains is not necessary for capsule production (Kroll \& Moxon, 1988), and strains with a deleted region in one repeat are still b+ (Ely et al., 1986; Kroll \& Moxon, 1988). We can now predict that such strains will show an altered pattern of capsule loss compared to the wild-type, and that different patterns will result from deletion of a cap DNA segment depending on whether it is located in the left or right repeat. The possibilities are illustrated in Fig. 4. In example 1, a deletion is located in the left repeat, $x$ $\mathrm{kb}$ from the left end. Recombinational reduction of cap between homologous regions in the left $x \mathrm{~kb}$ will result in a regular class I phenotype, but in the right $y \mathrm{~kb}$ will result in a residual locus which retains the deletion and is therefore at a stroke defective in both polysaccharide synthesis and export and thus has the class II phenotype. If, as shown, $x<<y$, and the simplifying observation is made that there are no 'hotspots' for recombination, the phenomenon observed macroscopically should be an unstable capsulate phenotype, readily giving rise to class II mutant colonies and only very rarely class I, as has indeed been found (Kroll \& Moxon, 1989). As a numerical example, with $x=3 \mathrm{~kb}$, the deletion $=1 \mathrm{~kb}$ and $y=13 \mathrm{~kb}$, class II colonies are predicted at $20 \%$ of $13 / 16=16 \cdot 3 \%$, while class I colonies are predicted at only $0.5 \%$ of $3 / 16=0.1 \%$. If the same deletion is located in the other repeat (Fig. 4, example 2) a very different situation is predicted. Now the genotype retaining the deletion is rare. With $x<<y$, the rate of production of class I colonies should be near normal. However, even a low rate of production of the class II genotype will translate into the same rate of class II colonial phenotype if there is no impediment to proliferation, so we can predict an unstable capsulate phenotype with both class I and class II mutant colonies, the latter albeit at a lower rate than in example 1. Taking the same values for $x$ and $y$, now class I colonies are predicted at $0.5 \%$ of $13 / 16=0 \cdot 4 \%$, but class II at $20 \%$ of $13 / 16=3.8 \%$. Such differences were observed by Ely et al. (1986), who speculated that the different apparent stabilities of the capsulate phenotype between members of such pairs of mutants might reflect functional differences between the two repeats in cap. While this is certainly possible, we now offer this alternative simpler explanation based on our better understanding of class I and II mutants.

In conclusion, the unexpectedly low rate with which Rec-mediated capsule loss appears to occur in $H$. influenzae type $\mathrm{b}$ can be explained by the greatly reduced viability of class I variants. Healthy capsule-deficient progeny only arise after a second 'rescue' mutation. The relative importance of class I and class II variants in the
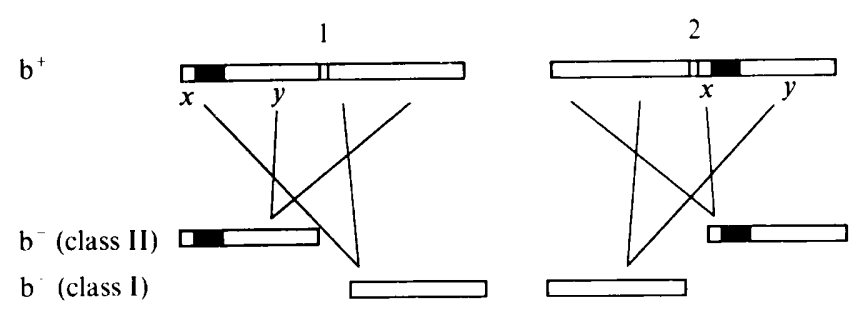

Fig. 4. Possible outcomes of recombinational reduction of cap in strains with asymmetric cap deletions. See text for details.

Haemophilus population is unknown. Class II variants are occasionally found among the non-typable Haemophilus that colonize the human respiratory tract. Such non-capsulate organisms appear to adhere better to human epithelial cells than their capsulate parents (St Geme \& Falkow, 1991). Thus, it may be that the 'bottleneck' that the class I phenotype imposes on the flow of organisms from capsulate to non-capsulate confers an advantage by ensuring that the population of capsulate organisms is not overwhelmed by capsuledeficient mutants, which cannot be restored to the capsulate phenotype except by transformation.

\section{References}

Alexander, H. E. \& Leidy, G. (1965). Determination of inherited traits of Haemophilus influenzae by deoxyribonucleic acid fractions isolated from type-specific cells. Journal of Experimental Medicine 93, 345-359.

ANDerson, P. W., Johnston, R. B., JK \& SMITH, D. H. (1972). Human serum activity against Haemophilus influenzae type b. Journal of Clinical Investigation 51, 31-38.

BUCKMIRE, F. L. A. (1976). Identification and quantitation of capsular antigen in capsulated and noncapsulated strains of Haemophilus influenzae type $\mathrm{b}$ by cross-immunoelectrophoresis. Infection and Immunity 13, 1733-1742.

BUCKMIRE, F. L. A. (1982). Characterisation of the capsular antigen isolated from Haemophilus influenzae type $\mathrm{b}$ and two spontaneous nonencapsulated mutants. In Haemophilus influenzae: Epidemiology, Immunology and Prevention of Disease, pp. 171-183. Edited by S. H. Sell \& P. F. Wright. New York: Elsevier/North-Holland Publishing Co.

Catlin, B. W. \& TARTAGni, V. R. (1969). Delayed multiplication of newly capsulated transformants of Haemophilus influenzae detected by immunofluorescence. Journal of General Microbiology 56, 387401.

Ely, S., TippetT, J., Kroll, J. S. \& Moxon, E. R. (1986). Mutations affecting expression and maintenance of genes encoding the serotype b capsule of Haemophilus influenzae. Journal of Bacteriology 167, 44-48.

Fothergill, L. D. \& Chandler, C. A. (1936). Observations on the dissociation of meningitic strains of Haemophilus influenzae. Journal of Immunology 31, 401-415.

Herriott, R. M., Meyer, E. M. \& Vogt, M. (1970). Defined nongrowth media for stage II development of competence in Haemophilus influenzae. Journal of Bacteriology 101, 517-524.

Hoiseth, S. K., ConNelly, C. J. \& Moxon, E. R. (1985). Genetics of spontaneous high-frequency loss of b capsule expression in Haemophilus influenzae. Infection and Immunity 49, 389-395. 
Hoiseth, S. K., Moxon, E. R. \& Silver, R. P. (1986). Genes involved in Haemophilus influenzae type b capsule expression are part of an $18 \mathrm{~kb}$ tandem duplication. Proceedings of the National Academy of Sciences of the United States of America 83, 1106-1110.

KROLL, J. S. \& MoXon, E. R. (1988). Capsulation and gene copy number at the cap locus of Haemophilus influenzae type b. Journal of Bacteriology 170, 859-864.

Kroll, J. S., Hopkins, I. \& Moxon, E. R. (1988). Capsule loss in Haemophilus influenzae type $\mathrm{b}$ occurs by recombination-mediated disruption of a gene essential for polysaccharide export. Cell 53, 347-356.
Moxon, E. R., Deich, R. A. \& Connelly, C. (1984). Cloning of chromosomal DNA from Haemophilus influenzae. Its use for studying the expression of type b capsule and virulence. Journal of Clinical Investigation 73, 298-306.

St GeME J. W., III \& FALKow, S. (1991). Loss of capsule expression by Haemophilus influenzae type $\mathrm{b}$ results in enhanced adherence to and invasion of human cells. Infection and Immunity 59, 1325-1333.

TURK, D. C. (1982). Clinical importance of Haemophilus influenzae. In Haemophilus influenzae: Epidemiology, Immunology and Prevention of Disease, pp. 3-9. Edited by S. H. Sell \& P. F. Wright. New York: Elsevier/North-Holland Publishing Co. 